\title{
Classification of focal liver lesions on ultrasound images by extracting hybrid textural features and using an artificial neural network
}

\author{
Yoo Na Hwang ${ }^{\mathrm{a}}$, Ju Hwan Lee ${ }^{\mathrm{b}}$, Ga Young Kim ${ }^{\mathrm{b}}$, Yuan Yuan Jiang ${ }^{\mathrm{b}, *}$ and Sung Min Kim ${ }^{\mathrm{a}, \mathrm{b},{ }^{\text {, }}}$ \\ ${ }^{a}$ Department of Medical Devices Industry, Dongguk University-Seoul, (100-715) 26, Pil-dong 3-ga, \\ Jung-gu, Seoul, South Korea \\ ${ }^{\mathrm{b} D e p a r t m e n t ~ o f ~ M e d i c a l ~ B i o t e c h n o l o g y, ~ D o n g g u k ~ U n i v e r s i t y-B i o ~ M e d i ~ C a m p u s, ~(410-820) ~ 32, ~}$ \\ Dongguk-ro, Ilsan Dong-gu, Goyang-si, Gyeonggi-do, South Korea
}

\begin{abstract}
This paper focuses on the improvement of the diagnostic accuracy of focal liver lesions by quantifying the key features of cysts, hemangiomas, and malignant lesions on ultrasound images. The focal liver lesions were divided into 29 cysts, 37 hemangiomas, and 33 malignancies. A total of 42 hybrid textural features that composed of 5 first order statistics, 18 gray level co-occurrence matrices, 18 Law's, and echogenicity were extracted. A total of 29 key features that were selected by principal component analysis were used as a set of inputs for a feed-forward neural network. For each lesion, the performance of the diagnosis was evaluated by using the positive predictive value, negative predictive value, sensitivity, specificity, and accuracy. The results of the experiment indicate that the proposed method exhibits great performance, a high diagnosis accuracy of over $96 \%$ among all focal liver lesion groups (cyst vs. hemangioma, cyst vs. malignant, and hemangioma vs. malignant) on ultrasound images. The accuracy was slightly increased when echogenicity was included in the optimal feature set. These results indicate that it is possible for the proposed method to be applied clinically.
\end{abstract}

Keywords: Ultrasound, focal liver lesions, classification, artificial neural network

\section{Introduction}

Ultrasound is extensively used to diagnose focal liver lesions since it provides an inexpensive and non-invasive means to visualize tissue characteristics. An ultrasound can provide real-time images of the lesion in which soft tissues can be differentiated. However, the classification accuracy of ultrasound imaging is relatively lower than that of computed tomography and magnetic resonance imaging due to the low resolution. In addition, it is difficult to diagnose focal liver lesions using only

\footnotetext{
* Address for correspondence: Yuan Yuan Jiang, Department of Medical Biotechnology, Dongguk University-Bio Medi Campus, (410-820) 32, Dongguk-ro, Ilsan Dong-gu, Goyang-si, Gyeonggi-do, South Korea. Tel.: +82-31-961-5158; Fax: +82-31-961-5350; E-mail: jiangyuanyuan86@gmail.com.

Sung Min Kim, Department of Medical Devices Industry, Dongguk University-Seoul, (100-715) 26, Pil-dong 3-ga, Junggu, Seoul, South Korea. Tel.: +82-31-961-5148; Fax:+82-31-961-5652; E-mail: smkim@dongguk.edu.
} 
an ultrasound image through a visual inspection due to the operator-dependent nature of the process [1-3].

The diagnostic accuracy can therefore be improved by implementing advanced computerized lesion differentiation via artificial neural network (ANN) and support vector machine (SVM) techniques. Such methods can extract clinical features from each of the different focal liver lesions, and these features provide a more accurate diagnosis through a computerized classification method that can process a medical image with greater accuracy than through visual inspection [4-6].

Poonguzhali, et al., [7] used principal component analysis (PCA) to determine the optimal feature group for focal liver lesions that could obtain an accuracy of $70 \%$ through a combination of GLCM and Law's features. Balasubramanian, et al., [8] classified focal liver lesions into normal, cyst, hemangioma, and malignant in liver ultrasound images using K-means and a back-propagation neural network (BPNN). BPNN was found to be suitable for the groups that were to be classified. Mittal, et al., [9] classified normal, cyst, hemangioma, and hepatocellular carcinoma according to a set of typical and atypical characteristics. Virmani, et al., [10] used a wavelet filter and a GA-SVM to classify normal, cirrhosis, and hepatocellular carcinoma on liver ultrasound images. Xian, et al., [11] reported a Fuzzy-SVM as an exercise tool to classify benign and malignant lesions in comparison with SVM. Jeon, et al., [12] suggested extracting multiple regions of interest (ROI) (ROI in, ROI out, ROI overlap, ROI posterior) for a suspicious lesion on ultrasound images in order to distinguish between focal liver lesions. In the present study, the method for multiple ROI obtained a higher accuracy than that acquired from a single ROI, however the accuracy in the classification between a malignant and hemangioma lesion was relatively low at $80 \%$.

The above studies indicated that the performance of diagnostic methods that use computer vision to assess lesions according to various characteristics are dependent upon the component of the feature set, the classifier, and the leaning algorithm [13, 14]. These previous studies suffer from two limitations. Firstly, the studies that have been performed thus far have focused much less on the diagnosis of various focal liver lesions on ultrasound images than on other disease areas. Conventional methods can obtain a meaningful accuracy in the differentiation between a cyst and hemangioma and between a cyst and a malignant lesion, nevertheless the accuracy in the differentiation between a hemangioma and a malignant lesion is still low due to the fact that these lesions present very similar tissue characteristics on ultrasound images.

The main purpose of this study therefore, is to improve the diagnostic accuracy of focal liver lesions by quantifying the key features for cysts, hemangiomas, and malignant lesions on ultrasound images and by evaluating the classification accuracy. To this end, information on the various characteristics of each of the different focal liver lesions is extracted and optimized. This present paper is organized as follows: Section II describes the feature extraction methods, the feature selection method, the artificial neural network, and the learning algorithm. Section III evaluates the performance of the proposed algorithm. Section IV presents the discussion, and Section V finally provides the conclusion.

\section{Materials and methods}

\subsection{Image acquisition}

This study was performed with 115 patients presenting with 99 focal liver lesions. The focal liver lesions were divided into 29 cysts, 37 hemangiomas, and 33 malignancies. The mean age of the patients was 58 years old, and the age range was from 29 to 78 years. All of the images were obtained 


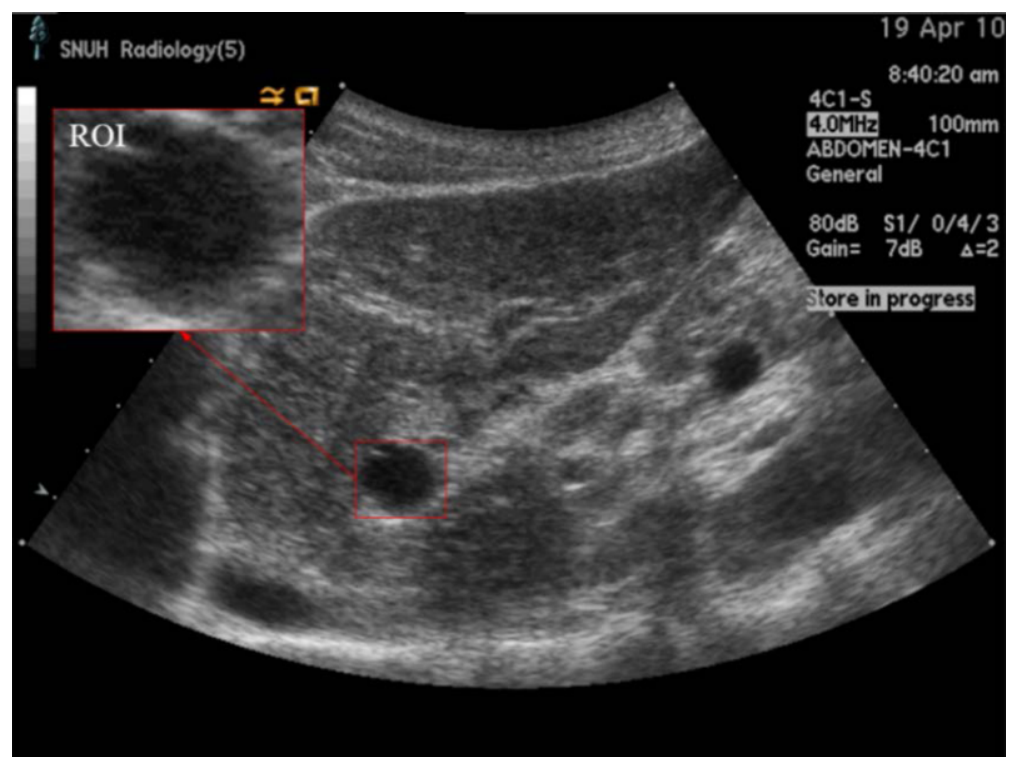

Fig. 1. Ultrasonic images of focal liver lesions, which were obtained using an Acuson Sequoia 512 ultrasound imager. The red rectangle shows the ROI that included the boundary of the lesions, and the size of the ROIs were $52 \times 52$ pixels $^{2}-$ $122 \times 157$ pixels $^{2}$.

with an Acuson Sequoia 512 ultrasound imager, under the approval of the Institutional Review Board of Seoul National University Hospital. The resolution for the gray level image was $640 \mathrm{x} 480$ pixels 2 . The transmitting frequency of the ultrasound was set to $2.0 \mathrm{MHz}$ and the receiving harmonic bandwidth was 4.0 MHz. The specific characteristics for each different lesion were obtained by selecting a ROI of $52 \times 52$ pixels $^{2}-122 \times 157$ pixels $^{2}$ from the original image. An experienced radiologist determined the ROI, which included the boundary of a focal liver lesion (Figure 1).

\subsection{Feature extraction}

The purpose of feature extraction is to improve the accuracy of the diagnosis by distinguishing the lesion area from a different area. In the present study, a total of 42 statistical textural features based on first order statistics, gray level co-occurrence matrix, Law's and echogenicity were obtained. The feature sets for each group are estimated as shown below. Figure 2 presents the entire image processing algorithm used to estimate the hybrid texture features from the original ultrasound images.

\subsubsection{First order statistics (FOS)}

The first-order histogram is used to extract five statistical features from the original image, as described in the following equations.

$$
\operatorname{Mean}\left(\mu_{i}\right)=\frac{\sum_{x=0}^{X-1} \sum_{Y=0}^{Y-1} I_{i}(x, y)}{X \times Y}
$$




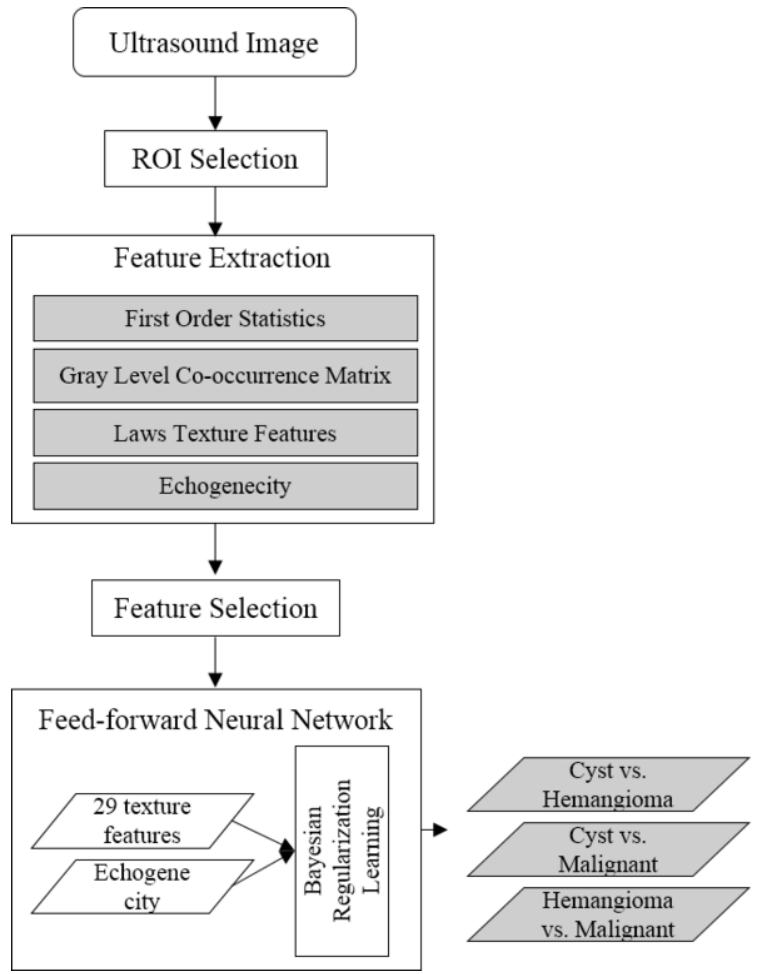

Fig. 2. Flow chart of the proposed algorithm for the classification of focal liver lesions.

$$
\begin{aligned}
& \text { Variance }=\frac{\sum_{x=0}^{X-1} \sum_{Y=0}^{Y-1}\left(I_{i}(x, y)-\mu\right)^{2}}{X \times Y} \\
& \text { Standard Deviation }\left(\sigma_{i}\right)=\sqrt{\frac{\sum_{x=0}^{X-1} \sum_{Y=0}^{Y-1}\left(I_{i}(x, y)-\mu\right)^{2}}{X \times Y}} \\
& \text { Skewness }= \\
& \text { Kurtosis }=\frac{\sum_{x=0}^{X-1 Y-1} \sum_{Y=0}\left(I_{i}(x, y)-\mu\right)^{3}}{X \times Y \times \sigma^{3}} \\
& \sum_{x=0}^{X-1} \sum_{Y=0}^{Y-1}\left(I_{i}(x, y)-\mu\right)^{4} \\
& X \times Y \times \sigma^{4}
\end{aligned}
$$

where $I_{i}(x, y)$ is the image intensity at point $(x, y)$, and $\mathrm{i}^{\text {th }}$ ROI image; $X$ and $Y$ denote the size of the ROI image; and $\mu$ is the mean of the ROI image and $\sigma$ is the standard deviation of the ROI image.

\subsubsection{Gray level co-occurrence matrix (GLCM)}

The GLCM is the second-order statistical approach that considers the relationship between the 
Table 1

A total of 18 features extracted from GLCM method

\begin{tabular}{l|l|l|l|l|l}
\hline \multicolumn{6}{l}{ GLCM } \\
\hline No. & Feature & No. & Feature & No. & Feature \\
\hline 1 & Energy & 7 & Autocorrelation & 13 & Sum Variance \\
\hline 2 & Entropy & 8 & Cluster Shade & 14 & Sum Entropy \\
\hline 3 & Dissimilarity & 9 & Cluster Prominence & 15 & Difference Variance \\
\hline 4 & Contrast & 10 & Maximum Probability & 16 & Difference Entropy \\
\hline 5 & Correlation & 11 & Sum of Squares & 17 & Information Measures of Correlation \\
\hline 6 & Homogeneity & 12 & Sum Average & 18 & Inverse Difference moment-Normalized \\
\hline
\end{tabular}

pixels [15]. The co-occurrence value $P\{(i, j) \mid(d, \theta)\}$ represents the frequency of an occurrence of a pair of gray-levels $(i, j)$ at a given distance $d$ with an orientation $\theta$. The present study used the unit distance and the $135^{\circ}$ orientation to reduce the computational load. The detailed algorithm for the GLCM was discussed by $[15,16]$. A total of 18 Haralick features are extracted from the co-occurrence matrices, as shown in Table 1.

\subsubsection{Law's texture features}

Law's features were used to extract energy information from the image. The Law's method [17-19] extracts texture energy measure features in three main steps. Firstly, five coefficient vectors were determined in order to represent level (L), edge (E), spot (S), ripple (R) and wave (W), as shown in Eq. (6), and a set of $5 \times 5$ kernels was formed by using each of the coefficient vectors.

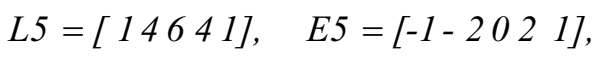

$$
\begin{aligned}
& S 5=\left[\begin{array}{lll}
-10 & 20-1]
\end{array}, R 5=[1-46-41]\right. \text {, } \\
& W 5=\left[\begin{array}{llll}
-1 & 2 & 0 & -2
\end{array}\right]
\end{aligned}
$$

Each 5 x 5 kernel was convolved with an ROI image. This step enabled the extraction of the specific characteristics of lesions by combining the original image with certain kernels. Rotational invariance is provided by generating each set of images from orthogonal matrices and then taking the average. A total of 9 energy maps can be resolved using Eq. (7).

$$
\begin{array}{ll}
\text { L5E5/E5L5 } & \text { L5S5/S5L5 } \\
\text { L5R5/R5L5 } & \text { E5E5 } \\
\text { E5S5/S5E5 } & \text { E5R5/R5E5 } \\
\text { S5S5 } & \text { S5R5/R5S5 } \\
\text { R5R5 } &
\end{array}
$$

According to [20], the features can be calculated from the above energy maps, and the sum of the absolute values/the number of pixels (SAV) or the sum of the squared values/the number of pixels (SSV) were the most significance statistics. Thus, these were comprised of a total of 18 Law's texture features by extracting SAV or SSV from each energy map, as shown in Eqs. (8) and (9). 


$$
\begin{aligned}
& S A V=\frac{\sum_{x=0}^{M-1} \sum_{Y=0}^{N-1}\left|R_{i}(x, y)\right|}{M \times N} \\
& S S V=\frac{\sum_{x=0}^{M-I N-1} \sum_{Y=0}^{N-1}\left(R_{i}(x, y)\right)^{2}}{M \times N}
\end{aligned}
$$

where $R_{i}(x, y)$ is the intensity of the convolution image at point $(x, y) ; M$ and $N$ denote the size of the ROI image.

\subsubsection{Echogenicity}

Echogenicity can extract the echoic characteristic of the lesions by comparing the gray scale value of the lesion inside with that of the lesion lateral based on pixel intensity. Echogenicity is one of the most useful clinical indices [21]. Echogenicity is calculated as the mean gray scale value of the ROI (inside) divided by that of the ROI (lateral), and its equation is given as [22]:

$$
\text { Echogenicity }=\frac{\text { Mean of the intensity }\left(\text { ROI }_{\text {inside }}\right)}{\text { Mean of the intensity }\left(\text { ROI Iateral }_{1}\right)}
$$

When the echogenicity value of the lesion is less than 0.9 , the lesion is determined to be hypoechoic, isoechoic between 0.9 and 1.1, and hyperechoic when greater than 1.1.

\subsection{Feature selection}

PCA with varimax rotation was performed to determine the optimal feature set. PCA can improve the classification accuracy and reduce computational time. This method transforms the feature vector by projecting the original data along the directions with greater variance. It is then possible to form an optimal feature set to classify the focal liver lesions, because the variance of the mean square error is reduced to an optimal value. PCA reduces the number of features used as input for the classifier, but the number of features that must be measured for classification is not actually reduced [23]. Since each extracted feature is usually a linear combination of the underlying features, a total of 29 optimum variables were selected from 42 original feature sets (Table 2).

\subsection{Classification}

Classification is the process during which the given input data is allocated to a predefined class in a manner that corresponds to the knowledge used to train the classifier. We used a two-layered feedforward neural network (FFNN) with a sigmoid function for classification. FFNN is widely used as a classifier for lesions since it has a simple structure but is robust against noise. FFNN is composed of 29 input neurons, 10 hidden neurons and 2 output neurons, as shown in Figure 3. A total of 29 key features that were selected by the PCA were used as a set of inputs for FFNN.

For training, a Bayesian regulation learning algorithm was applied to reduce over-fitting. This updates the weights and bias variables according to the Levenberg-Marquardt optimization. The connection weight vectors were randomly assigned at the beginning, and were continually modified to 
Table 2

The optimal features selected by PCA from 42 original features

\begin{tabular}{|c|c|c|}
\hline $\begin{array}{l}\text { Feature } \\
\text { Extraction } \\
\text { Method }\end{array}$ & No. of Features & Optimal Features \\
\hline FOS & 1 & Mean \\
\hline \multirow[t]{10}{*}{ GLCM } & \multirow[t]{10}{*}{10} & Autocorrelation \\
\hline & & Contrast \\
\hline & & Dissimilarity \\
\hline & & Entropy \\
\hline & & Sum of Squares \\
\hline & & Sum Average \\
\hline & & Sum Variance \\
\hline & & Sum Entropy \\
\hline & & Difference Variance \\
\hline & & Difference Entropy \\
\hline \multirow{18}{*}{$\begin{array}{l}\text { Laws Textural } \\
\text { Features }\end{array}$} & \multirow[t]{18}{*}{18} & E5'L5 \\
\hline & & S5'L5 \\
\hline & & E5'E5 \\
\hline & & S5'E5 \\
\hline & & R5'L5 \\
\hline & & R5'E5 \\
\hline & & S5’S5 \\
\hline & & R5'S5 \\
\hline & & R5'R5 \\
\hline & & E5'L5_2 \\
\hline & & S5'L5_2 \\
\hline & & E5'E5_2 \\
\hline & & S5'E5_2 \\
\hline & & R5'L5_2 \\
\hline & & R5'E5 2 \\
\hline & & S5'S5 2 \\
\hline & & R5'S5_2 \\
\hline & & R5'R5 2 \\
\hline
\end{tabular}

Note: Features from E5'L5 to R5'R5 are variables for SAV corresponding to each of the different masks. Features from E5'L5_2 to R5'R5_2 are variables for SSV.

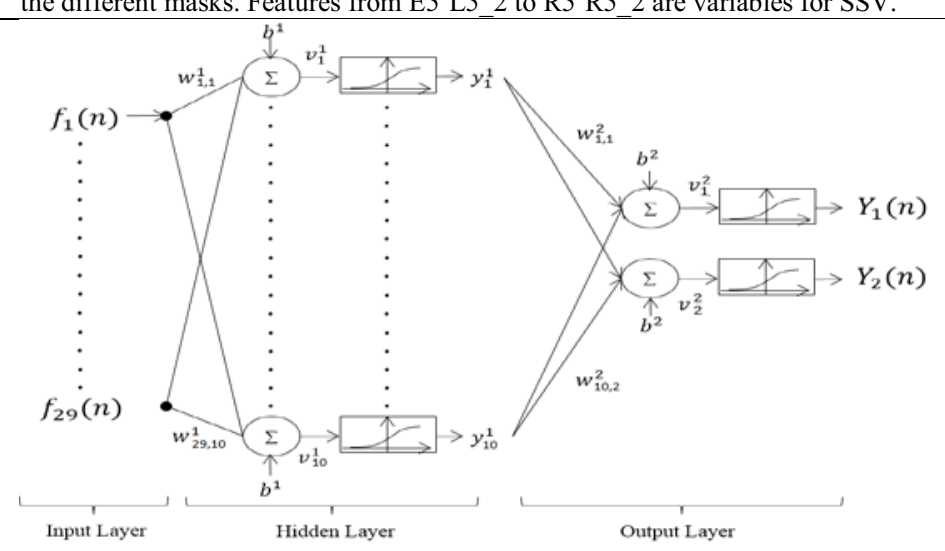

Fig. 3. Structure of the feed-forward neural network composed of one input layer, one hidden layer, and one output layer. 
reduce the overall system error. It is possible to minimize the combination of the squared error and the weights as well as to determine the right combination to form a well-generalized network [24-26]. In addition, it can measure a mean square error and a percentage error, and this process can be used to classify the values of the input variables into three groups corresponding to cysts, hemangiomas, and malignant lesions.

\subsection{Performance evaluation}

For each lesion, the performance of the diagnosis was evaluated using the positive predictive value (PPV), negative predictive value (NPV), sensitivity, specificity and accuracy based on the true positive (TP), true negative (TN), false positive (FP) and false negative (FN) rates.

$$
\begin{gathered}
P P V=\frac{(T P)}{(T P+F P)} \\
N P V=\frac{(T N)}{(T N+F N)} \\
\text { Sensitivity }=\frac{(T P)}{(T P+F N)} \\
\text { Specificity }=\frac{(T N)}{(T N+F P)} \\
\text { Accuracy }=\frac{(T P+T N)}{(T P+T N+F P+F N)}
\end{gathered}
$$

where $T P$ is the number of 'malignant' lesions that are correctly categorized as 'malignant', $T N$ is the number of 'non-malignant' lesions that are correctly categorized as 'non-malignant'. $F P$ is the number of 'non-malignant' lesions that are misclassified as 'malignant'. $F N$ is the number of 'malignant' lesions that are misclassified as 'non-malignant'. 'Non-malignant' denotes other lesions (cysts and hemangiomas) that are classified differently from malignant ones. Thus, the hemangioma lesion was designated as a 'malignant' type during classification between a cyst and a hemangioma lesion. $P P V$ is the percentage of predictive positives that are actually positive, and $N P V$ is the percentage of predictive negatives that are actually negative. The sensitivity is the ability for the classification model to classify malignant cases, the specificity is the ability to identify benign cases, and the accuracy is the ability to diagnose all cases.

The accuracy of the diagnosis for the lesion was estimated using the receiver operating characteristic (ROC) curve. The ROC analysis was performed in order to show the efficiency of the trade-off between the $T P$ and the $F P$ [27]. The performance of the diagnosis was evaluated in terms of the area under the ROC curve (AUC), where a higher AUC indicates a higher accuracy.

\section{Experimental results}

\subsection{Comparison of diagnosis accuracy in accordance with each different PCA component}


The accuracy is compared according to diverse feature sets, and the three groups are determined by conducting a PCA to evaluate the classification accuracy of each group. Table 2 demonstrates a total of 29 features composed of FOS, GLCM, Law's, and echogenicity optimized by PCA selection.

When feature set 1 was selected as the optimal feature group, the accuracy of the diagnosis between a cyst and a hemangioma as well as between a cyst and a malignant lesion was $97.72 \%$ and $97.63 \%$, respectively. These results were slightly improved by applying feature set 2 . To classify between hemangioma and a malignant lesion, the accuracy of the diagnosis with feature set 2 largely increased by $22.14 \%$ when compared with feature set 1 , nevertheless it still showed a very low accuracy of $72.72 \%$. However, for classification between a hemangioma and a malignant lesion, feature set 3 resulted in an accuracy that was greatly increased by $18.44 \%$, although it did not show a large difference relative to the accuracy of feature set 2 when classifying between a cyst and a hemangioma as well as between a cyst and a malignant lesion. More importantly, feature set 3 showed a high diagnosis accuracy of over $90 \%$ among all classification groups (cyst vs hemangioma, cyst vs malignant, and hemangioma vs malignant) (Tables 3-5).

Table 3

Classification results between cysts and hemangioma lesions according to the different feature sets determined by PCA

\begin{tabular}{l|l|l|l|l|l|l}
\hline \multirow{2}{*}{ Group } & \multicolumn{5}{l}{ Cyst vs. Hemangioma } & \multicolumn{4}{l}{} \\
\cline { 2 - 7 } & PPV & NPV & Sensitivity (\%) & Specificity (\%) & Accuracy (\%) & AUC \\
\hline Feature set 1 & 97.3 & 96.55 & 97.84 & 97.67 & 97.72 & 0.976 \\
\hline Feature set 2 & 97.3 & 96.55 & 97.84 & 97.95 & 97.9 & 0.977 \\
\hline Feature set 3 & 97.37 & 100 & 98.11 & 98.64 & 98.35 & 0.986 \\
\hline
\end{tabular}

Table 4

Classification results between cysts and malignant lesions according to the different feature sets determined by PCA

\begin{tabular}{l|l|l|l|l|l|l}
\hline \multirow{2}{*}{ Group } & \multicolumn{6}{l}{ Cyst vs. Malignant } \\
\cline { 2 - 7 } & PPV & NPV & Sensitivity (\%) & Specificity (\%) & Accuracy (\%) & AUC \\
\hline Feature set 1 & 100 & 96.67 & 96.96 & 98.3 & 97.63 & 0.976 \\
\hline Feature set 2 & 97.06 & 100 & 99.09 & 97.95 & 98.56 & 0.985 \\
\hline Feature set 3 & 96.97 & 96.55 & 98.4 & 97.95 & 98.24 & 0.982 \\
\hline
\end{tabular}

Table 5

Classification results between hemangiomas and malignant lesions according to the different feature sets determined by PCA

\begin{tabular}{l|l|l|l|l|l|l}
\hline \multirow{2}{*}{ Group } & \multicolumn{7}{l}{ Hemangioma vs. Malignant } & \multicolumn{4}{l}{} \\
\cline { 2 - 7 } & PPV & NPV & Sensitivity (\%) & Specificity (\%) & Accuracy (\%) & AUC \\
\hline Feature set 1 & 46.42 & 68.75 & 40 & 60 & 50.58 & 0.5 \\
\hline Feature set 2 & 73.33 & 72.5 & 67.59 & 77.3 & 72.72 & 0.726 \\
\hline Feature set 3 & 86.11 & 94.12 & 95.14 & 86.68 & 91.16 & 0.909 \\
\hline
\end{tabular}

Table 6

Classification result with and without echogenicity in feature set 3 according to each of the different classification groups

\begin{tabular}{l|l|l|l|l|l|l}
\hline Group & PPV & NPV & Sensitivity (\%) & Specificity (\%) & Accuracy (\%) & AUC \\
\hline $\begin{array}{l}\text { Cyst vs. } \\
\text { Hemangioma }\end{array}$ & $99.37 / 100$ & $100 / 100$ & $98.11 / 100$ & $98.64 / 99.32$ & $98.35 / 99.7$ & $0.986 / 0.997$ \\
\hline
\end{tabular}




\begin{tabular}{l|l|l|l|l|l|l}
\hline $\begin{array}{l}\text { Cyst vs. } \\
\text { Malignant }\end{array}$ & $96.97 / 100$ & $96.55 / 100$ & $98.4 / 98.79$ & $97.95 / 98.64$ & $98.24 / 98.72$ & $0.982 / 0.987$ \\
$\begin{array}{l}\text { Hemangioma vs. } \\
\text { Malignant }\end{array}$ & $86.11 / 94.12$ & $94.12 / 97.22$ & $95.14 / 98.2$ & $86.68 / 94.33$ & $91.16 / 96.13$ & $0.909 / 0.963$ \\
\hline
\end{tabular}

Table 7

Comparison of the diagnosis accuracy between the preliminary study and proposed method

\begin{tabular}{l|l|l|l}
\hline Group & Cyst vs. Hemangioma (\%) & Cyst vs. Malignant (\%) & Hemangioma vs. Malignant (\%) \\
\hline Previous study [10] & 96.5 & 95.8 & 80 \\
\hline The present study & 99.7 & 98.72 & 96.13 \\
\hline
\end{tabular}

3.2. Comparative result of the performance evaluation depending on the inclusion/exclusion of the echogenicity from feature set 3

Although echogenicity was excluded from the feature set during feature selection, this feature has been used clinically. Therefore, the performance was evaluated with and without echogenicity in feature set 3. Table 6 shows a comparison of the performance between feature set 3 (excluding echogenicity) and feature set 3 (E), which included echogenicity.

In feature set 3 , the accuracy of the diagnosis for cyst vs. hemangioma and cyst vs. malignant slightly increased by $1.35 \%$ and $0.48 \%$, respectively. In contrast, the classification for hemangioma vs. malignant increased considerably not only in terms of accuracy by $5 \%$, but also in terms of sensitivity, specificity, and AUC. The PPV distinguishing a malignant lesion from a hemangioma was less than $90 \%$ in feature set 3 , at $86.11 \%$. Feature set 3 (E) offered a significant improvement of over $8 \%$, with a PPV of $94.12 \%$. These results therefore indicate that echogenicity improved the performance of the diagnosis of focal liver lesions.

\subsection{Comparison with our previous study}

In order to evaluate whether the accuracy of the proposed method improved, a comparative study was performed between the proposed method and that of our preliminary study. The preliminary study had shown meaningful diagnosis accuracy for the classification of cyst vs. hemangioma and cyst vs. malignant at $96.5 \%$ and $95.8 \%$, respectively, using a multiple ROI selection. However, the classification for hemangioma vs. malignant had shown a low accuracy [12]. The proposed method exhibits a superior accuracy for all classification groups when compared with that of our preliminary study. In particular, a considerable improvement was observed when classifying hemangioma vs. malignant, with an accuracy of $96.13 \%$. Table 7 shows a comparison of the classification accuracy between the proposed feature set 3 (E) and that of the preliminary study [12], in accordance with each group.

\section{Discussion}

The amount of information according to the feature sets used in the PCA followed a relationship where feature set $3>$ feature set $2>$ feature set 1 . Although feature set 1 and feature set 2 had relatively little information, these showed a high accuracy of $97 \%$ for classifying cyst vs. hemangioma and also for cyst vs. malignant. However, for hemangioma vs. malignant, they showed a very low accuracy of $50.58 \%$ and $72.72 \%$, respectively. This is due to the similarity of each of the lesions. In 
the ultrasound images, cysts commonly show distinct characteristics including anechoic behavior, enhancement of posterior acoustics, and a clear boundary of the lesion when compared to that of other lesions, such as hemangiomas or malignant lesions. Thus, it is possible for cysts to be differentiated from hemangiomas and malignant lesions with a small amount of information. Conversely, it is difficult for hemangiomas to be differentiated from malignant lesions because the larger the hemangioma area is, the more irregular the boundary, and the lesion has similar characteristics to a malignant lesion in that they both have a hypoechoic or mixed echoic pattern [21,28]. For this reason, more information is required to distinguish a hemangioma from a malignant lesion than when differentiating between a cyst and a hemangioma and between a cyst and a malignant lesion. This study shows important results for all focal liver lesions by optimizing the feature set.

During feature selection, echogenicity was not selected in the PCA, but echogenicity is often used clinically because different echoic behaviors are presented by each variable focal liver lesion. Thus, the diagnosis accuracy was evaluated according to whether echogenicity was included or excluded from feature set 3 . Although the results provided by the existing feature set 3 were adequate, the accuracy was slightly increased when echogenicity was included in the optimal feature set. In particular, the classification of hemangioma vs. malignant showed an improved accuracy because the echoic behavior is shown, unlike in other features that consider the texture of the lesion on the inside only. A cyst is commonly anechoic with a thin wall and enhanced posterior acoustics, but a hemangioma typically has a fine, hyperechoic nature $(<2 \mathrm{~cm})$. A malignant lesion usually has a hypoechoic nature but has a variable acoustic mass by size. At less than $1 \mathrm{~cm}$ in diameter, $92 \%$ of malignant lesions are hypoechoic, and for a diameter of less than $2 \mathrm{~cm}$, this figure reduces to $60-80 \%$ [29]. When the mass is larger than $1.5 \mathrm{~cm}$, a clear moon halo shows that the periphery of the mass is more hypoechoic than the center of the mass, and this characteristic can be used to classify the focal liver lesions in the present study. Echogenicity is also found to be an important feature for the classification of focal liver lesions, and in addition, the best performance is not always achieved when the feature set is selected via PCA. Therefore, the selection of the feature set should be performed with practical considerations.

The proposed method exhibits an improved classification for focal liver lesions. Our preliminary study compares the accuracy with multiple ROIs (ROI in, ROI out, ROI overlap and ROI posterior) in order to classify focal liver lesions (cysts, hemangiomas, and malignant lesions). In the comparison, results show that multiple ROI had the best accuracy. A high accuracy was observed when classifying cysts vs. hemangiomas and also cysts vs. malignant lesions, but an accuracy of $80 \%$ was only achieved when classifying hemangiomas vs. malignant lesions. The proposed method exhibits excellent classification performance using an optimized feature set even though the proposed method applies a single ROI extraction. The results indicate that the determination of the optimal feature set is more important than the selection of the ROI. In addition, our preliminary study used an SVM based on an RBF kernel. However, when dealing with highly nonlinear and complex systems, like with the ultrasound systems, ANNs are generally superior to SVMs because many hidden layers, nodes, and parameters can be easily adjusted in an ANN. The proposed method shows excellent performance in classification of focal liver lesions when compared with the method described in our preliminary study.

\section{Conclusions}

The results of the experiment indicate that the proposed method exhibits great performance in terms 
of classifying focal liver lesions on ultrasound images. The classification to distinguish hemangiomas from malignant lesions shows meaningful results. In addition, echogenicity was found to be a key feature in the classification of focal liver lesions. These results indicate that it is possible for the proposed method to be applied clinically. However, despite its superior performance, a limitation of the proposed method is that a small number of subjects were included in the experiment. Therefore, a large number of subjects should be included to study the classification of variable focal liver lesions with a high diagnosis accuracy.

\section{Acknowledgment}

This work was supported by International Collaborative R \& D Program funded by the Ministry of Knowledge Economy (MKE), Korea. (N01150049, Developing high frequency bandwidth (40-60 $\mathrm{MHz}$ ) high resolution image system and probe technology for diagnosing cardiovascular lesion)

\section{References}

[1] W.L. Lee, An ensemble-based data fusion approach for characterizing ultrasonic liver tissue, Applied Soft Computing 13 (2013), 3683-3692.

[2] R. Suganya and S. Rajaram, Classification of liver diseases from ultrasound images using a hybrid Kohonen SOM and LPND speckle reduction method, Int. Conf. Signal Processing, Computing and Control (ISPCC) IEEE, Waknaghat Solan, 2012, pp. 1-6.

[3] S.A. Azaid, M.W. Fakhr and A.F.A. Mohamed, Automatic diagnosis of liver diseases from ultrasound images, Proc. Int. Conf. Computer Engineering and Syst. (ICCES) IEEE, Cairo, 2006, pp. 313-319.

[4] H. Sujana, S. Swarnamani and S. Suresh, Application of artificial neural networks for the classification of liver lesions by image texture parameters, Ultrasound in Medicine \& Biology 22 (1996), 1177-1181.

[5] A.A. Sakr, M.E. Fares and M. Ramadan, Automated focal liver lesion staging classification based on Haralick texture features and multi-SVM, International Journal of Computer Applications 91 (2014), 17-25.

[6] K. Wu, X. Chen and M. Ding, Deep learning based classification of focal liver lesions with contrast-enhanced ultrasound, Optik 125 (2014), 4057-4063.

[7] S. Pooguzhali, B. Deepalaskhmi and G. Ravindran, Optimal feature selection and automatic classification of abnormal masses in ultrasound liver images, IEEE Int. Conf. Sign. Processing, Communications and Netw, Chennai, 2007, pp. 503-506.

[8] B. Deepalaskhmi, S. Pooguzhali and G. Ravindran, Automatic classification of focal lesions in ultrasound liver images using principal component analysis and neural networks, Proc. Int. Conf. IEEE EMBS, Lyon, 2007, pp. 2134-2137.

[9] D. Mittal, V. Kumar and S.C. Saxena, et al., Neural network based focal liver lesion diagnosis using ultrasound images, Computerized Medical Imaging and Graphic 35 (2011), 315-323.

[10] J. Virmani, V. Kumar, N. Kalra and N. Khandelwal, SVM-based characterization of liver ultrasound images using wavelet packet texture descriptors, Journal of Digital Imaging 26 (2013), 530-543.

[11] G. Xian, An identification method of malignant and benign liver tumors from ultrasonography based on GLCM texture features and fuzzy SVM, Expert Systems with Applications 37 (2010), 6737-6747.

[12] J.H. Jeon, J.Y. Choi, S.H. Lee and Y.M. Ro, Multiple ROI selection based focal liver lesion classification in ultrasound images, Expert Systems with Applications 40 (2013), 450-457.

[13] J. Virmani, V. Kumar, N. Kalra and N. Khandelwal, A comparative study of computer-aided classification systems for focal hepatic lesions from B-mode ultrasound, Journal of Medical Engineering \& Technology 37 (2013), $292-306$.

[14] K.R. Krishnan and R. Sudhakar, Automatic classification of liver diseases from ultrasound images using GLRLM texture features, Soft Computing Applications AISC 195 (2013), 611-624.

[15] R.M. Haralick, K. Shanmugam and I. Dinestein, Textural features for image classification, IEEE Transactions on Systems, Man and Cybernetics 3 (1973), 610-621.

[16] L. Soh and C. Tsatsoulis, Texture analysis of SAR sea ice imagery using gray level Co-occurrence matrices, IEEE Transaction on Geoscience and Remote Sensing 37 (1999), 780-795.

[17] K.I. Laws, Texture image segmentation, Ph.D. Dissertation, Image Processing Institute, University of Southern 
California, 1980.

[18] K.I. Laws, Rapid texture identification, Proc. SPIE Image Processing for Missile Guidance 238 (1980), 376-380.

[19] L.G. Shapiro and G.C. Stockman, Computer Vision, 1st ed., Prentice Hall, New Jersey, 2001.

[20] P. Matti and A. Rosenfeild, Experiments with texture classification using averages of local pattern matches, IEEE Transactions on Systems, Man, and Cybernetics SMC 13 (1983), 421-426.

[21] C.J. Harvey and T. Albrecht, Ultrasound of focal liver lesions, European Radiology 11 (2001), 1578-1593.

[22] S.H. Kim, M.L. Jeong and G.K. Kwang, et al., Computer-aided image analysis of focal hepatic lesions in ultrasonography: Preliminary results, Abdominal Imaging 34 (2009), 183-191.

[23] A.P. Nanthagopal and R.S. Rajamony, Automatic classification of brain computed tomography images using waveletbased statistical texture features, Journal of visualization 15 (2012), 363-372.

[24] S. Haykin, Neural Networks: A Comprehensive Foundation, 2nd ed., Prentice Hall, New Jersey, 2002.

[25] D. MacKay, Bayesian interpolation, Neural Computation 4 (1992), 415-447.

[26] R. Gencay and Qi Min, Pricing and hedging derivative securities with neural networks: Bayesian regularization, early stopping, and bagging, IEEE Transaction on Neural Networks 12 (2001), 726-734.

[27] J.R. Beck and E.K. Shultz, The use of relative operating characteristic (ROC) curves in test performance evaluation, Archives of Pathology \& Laboratory Medicine 110 (1986), 13-20.

[28] Q. Emilio, C. Fabrizio and M. Bertolotto, et al., Characterization of focal liver lesions with contrast-specific US modes and a sulfur hexafluoride-filled microbubble contrast agent, Diagnostic Performance and Confidence 232 (2004), 420430.

[29] R. Badea and S. Ioanitescu, Ultrasound imaging of liver tumors-current clinical applications, in: Liver Tumors, A. Julianov, ed., InTech, Croatia, 2012, pp. 75-102. 